\title{
LA GIULLERIA IN ITALIA. PRATICA SCENICA E FESTIVA
}

\author{
Tito Saffioti \\ Periodista i escriptor \\ info@titosaffioti.it
}

\begin{abstract}
Resum
Che la giulleria italiana abbia avuto una sua specificità rispetto a quella di altre aree europee è domanda alla quale è difficile dare una risposta. In realtà i frequenti scambi culturali fra i giullari di aree diverse erano favoriti dal loro nomadismo. L'autore si sofferma ad esaminare i contatti intercorsi tra l'Italia e la Spagna, citando esempi che dimostrano la diffusa presenza di intrattenitori spagnoli in Italia e di italiani in terra iberica.

La vita dei buffoni di corte italiani era assai differente da quella dei francesi, che spesso erano assunti en titre d'office e dunque avevano a corte un ruolo riconosciuto. In Italia essi dovevano industriarsi tra vari incarichi e non disdegnavano neanche quelli più infami: uno di loro si offre perfino di eseguire la sentenza che condanna un prete alla castrazione.

C'erano tuttavia anche in Italia ruoli ufficializzati e molto ambiti: erano quelli degli araldi e canterini che venivano assunti dalle amministrazioni comunali di molte città dell'Italia centrale e che avevano il compito di rallegrare i reggitori della Cosa pubblica nelle pause del loro lavoro. Un'altra importante occasione di lavoro si offrirà poi con la rinascita dell'istituzione teatrale, che prenderà il via nell'Italia umanistica e che avrà in loro importanti punti di riferimento.
\end{abstract}

\section{Paraules clau}

Joglaria italiana, bufons de cort, heralds, nomadisme joglaresc, iconografia tardomedieval i renaixentista

\begin{abstract}
A difficult question to answer is whether the Italian giulleria had its own specificity compared to that of other European areas. In fact, the frequent cultural exchanges among the different areas of jesters were encouraged by their nomadic lifestyle. The author pauses to examine the contacts between Italy and Spain, citing examples that demonstrate the widespread presence of Spanish entertainers in Italy and Italians in Iberia.

The life of the Italian court jesters was very different from that of the French ones, who were often recruited en titre d'office and therefore had a recognized role at court. In Italy they had to strive in the various tasks and did not disdain even the most infamous
\end{abstract}


one: one of them even offers to execute the sentence which condemns a priest to castration.

In Italy, however, there were also formalized and sought-after roles: those were the heralds and singers who were hired by local government of many cities in central Italy and had the task of cheering the leaders of res publica in the pauses of their work. Another important job opportunity will then be played with the rebirth of the theatrical institution, that will start in the humanistic Italy and which will have in them important benchmarks.

\section{Keywords}

Italian minstrels, court jesters, heralds, nomadic minstrels, Late Medieval and Renaissance iconography

\section{5}

Che la giulleria italiana possieda una sua specificità rispetto a quella di altre aree europee, è tesi piuttosto controversa e soltanto in parte vera. Come ho cercato di dimostrare in un mio recente volume, ${ }^{\mathrm{I}}$ in realtà tutti i popoli e tutte le epoche hanno avuto figure di intrattenitori professionisti in qualche misura assimilabili al nostro "giullare".

Tuttavia, il loro nomadismo li ha portati ad avere frequenti e numerosi scambi con le nazioni limitrofe e ciò ha creato un terreno comune, all'interno del quale è difficile distinguere quanto di autoctono era in loro e quanto di acquisito. Questo raccontino testimonia assai bene di come fossero continui e proficui i rapporti tra giullari di varie nazionalità anche al fine, come nel caso che segue, di accrescere la conoscenza di trucchi del mestiere:

Histriones duo quorum unus Anglicus qui veniebat in Franciam addiscere subtilitates, alius Gallicus qui ibat in Angliam addiscere subtilitates, oviaverunt sibi. Et querentes invicem causas itineris sui, dixit Anglicus: - Scio quicquid est in Anglia — et alius: - Scio quicquid est in Francia; exerceamus hic subtilitates et non procedamus_- ${ }^{2}$

I Cfr. T. SAfFioti, Gli occhi della follia. Giullari e buffoni di corte nella storia e nell'arte, Milano, 2009, specificatamente il cap. v, pp. 4I-58.

2 "Due istrioni, uno inglese che andava in Francia per imparare delle subtilitates e l'altro francese che andava in Inghilterra per imparare delle subtilitates, si incontrarono [a mezza strada]. E, essendosi interrogati a vicenda sui motivi che li avevano spinti a compiere il viaggio, l'inglese disse: - Io so tutto quello che c'è [da imparare] in Inghilterra-. Rispose l'altro: - Io so tutto quello che c'è [da imparare] in Francia; mettiamo a confronto le nostre subtilitates e non andiamo oltre-". Cfr. A. Hilka, "Vermichtes zu den mittelalterlichen Vaganten, Gauklern und Gelegenheitsdichtern", Studi Medievali, n. s., n. 2, (1929), p. 422, n. I5. 
Già che sono ospite di questa meravigliosa terra, mi pare doveroso soffermarmi brevemente sugli scambi avvenuti tra i nostri due Paesi. Il giullare spagnolo di religione ebraica Juan de Valladolid, nel I458 era di passaggio a Ferrara, dove si mischiò con i numerosi suoi colleghi che volteggiavano alla corte di Borso d'Este. A questi egli chiese una lettera di presentazione per il duca di Milano, che prontamente ottenne, anche se il tono non appare particolarmente entusiastico:

Lo exhibitore presente chiamato Zoan de Vagliadolid poeta hispano et vulgare, secundo lui, ne ha pregato cum instantia che nuy el vogliamo racomendare ala Vostra Excellentia. Et perché luy dice essere huomo et cortegiano de la Maiestà del re de Ragona et di Navara etc., et anche pare che pur il sapia dir in rima, rogati lo raccomandemo ala V. Celsitudine que bene valeat et nos amet".

Nel corso del suo viaggio, Juan si fermò alcuni giorni alla corte di Mantova, dove riuscì a strappare un'altra commendatizia al marchese Ludovico Gonzaga. Pare che a Milano egli abbia ricevuto buona accoglienza da parte di Francesco Sforza, tanto più che si spacciò per astrologo, una qualifica che in ogni tempo ottiene spropositata considerazione. Nel 1462 assume una nuova, altrettanto improbabile, specializzazione e torna a Mantova come "incantatore della grandine" accompagnato, naturalmente, da una lettera di presentazione dello Sforza. Nel I473 lo vediamo installato alla corte degli Aragona a Napoli, dopodiché torna in patria e precisamente a Siviglia, presso Isabella la Cattolica.

Un altro curioso episodio ha per protagonista un papa nato non molto distante da qui, Rodrigo Borgia, che resse il pontificato col nome di Alessandro VI dal I492 al I503. Alla sua corte non dovevano mancare nani e buffoni provenienti dalla sua terra d'origine, come è il caso di tale Gabrieletto. Questa scena che lo vede in azione è descritta dal cerimoniere Burcardo in un latino così saporoso - e comprensibile — che non ha bisogno di traduzione, basterà solo aggiungere che la qui citata camera papagalli è una stanza degli appartamenti vaticani dove si teneva il concistoro segreto:

Gabrielletus bufo, papa exeunte de loco benedictionis predicto, finxit continue predicare latine et hispanice, alta, clamorosa voce, usque ad cameram paramenti; cum in camera papagalli papa deponeret paramenta, dixit se optime cantasse, de quo gloriabatur. ${ }^{4}$

3 "Il latore della presente, chiamato Zoan de Vagliadolid, che si dichiara poeta in lingua spagnola e italiana, ci ha pregato con insistenza di volerlo raccomandare a vostra eccellenza. Egli afferma di essere cortigiano di sua maestà il re di Aragona e di Navarra etc., e pare anche che sappia improvvisare testi in rima. Dietro sua richiesta dunque lo raccomandiamo alla vostra celsitudine, nella speranza che stiate bene e ci conserviate il vostro affetto". Cfr. E. MоттA, "Giovanni de Valladolid alle corti di Mantova e di Milano (I458-I473)”, Archivio Storico Lombardo, s. II, a. XVIII, (I980), p. 938.

4 Cfr. J. Burckardi, Liber Notarum ab anno MCCCLXXXIII usque ad annum MDVI, ed. Eugenio 
Tra il 1307 e il I3I9 giungono alla corte d'Aragona di Giacomo II il Giusto i giullari italiani Albertino da Genova, Nicoluzzo, Veneziano da Messina, Bussiquello e Francesco (questi due giullari degli ambasciatori pisani), e Giorgio, proveniente dalla Sicilia. Nel 1548 il nano-buffone Gradasso, che qui vediamo in tutto il trionfo della sua possanza, [Fig. I] giunse alla corte di Filippo d'Austria recando alcuni cavalli donati dal marchese del Vasto. Allo scopo di accudirli, egli alloggiava all'interno della scuderia. ${ }^{5}$

Una delle più antiche testimonianze sui giullari italiani di cui ho trovato traccia, riguarda un ciarlatano italiano che faceva straordinari giochi di prestigio con l'aiuto di un cane ammaestrato, è descritto con vividi tratti nella Historia Romana di Landolfo Sagace. Siamo a Costantinopoli nell'anno 543 d. C., dove

apparuit quidam ex regione Italorum per uillas discurrens, nomine Andreas, habens secum canem rufum et cecum, qui iussus ab eo faciebat miracula. Cum enim staret ipse in foro et turba in circuitu edesset, clam eodem cane deferebantur astantium anuli aurei et argenti ac ferrei et ponebantur in pauimento, quos ille cooperiebat et precibiebat cani; et tollebat et dabat unicuique suum. Similiter et diuersorum imperatorum numismata mixta porrigebat per nomina. Sed presto turba astante, uirorum scilicet ac mulierum, interrogatus ostendebat in utero habentes et fornicarios et adulteros et auarios et magnanimos. $^{6}$

Dopo aver notato per inciso che chiedere al cane di riconoscere i generosi, era nulla più che un furbo espediente per mettere i prescelti nell'obbligo di dimostrarsi tali, passiamo oltre. Da noi i buffoni non erano regolarmente retribuiti come avveniva in Francia con i bouffons en titre d'office. La loro funzione molto di rado si limitava all'intrattenimento, anzi essi potevano essere impiegati in servizi di ogni genere, occasionalmente fungevano da scalchi, camerieri, galoppini e altre attività ancora più umili. Questa indeterminatezza della figura del buffone in Italia va sempre tenuta presente, incerti come erano tra mille mestieri che soltanto

Celani, vol. II, L. A. Muratori, Rerum Italicarum Scriptores², t. XxxiI, p. I, Città di Castello, I9II, p. 275 .

5 Cfr. F. J. Bouza Alvarez, Locos, enanos i hombre de placer en la corte de los Austrias. Oficio de burlas, Madrid, $1996^{2}$, p. 169.

6 "Un tale, di nome Andrea, venne da contrade italiche portando con sé un cane fulvo e cieco che, dietro suo ordine e a un suo cenno si produceva in mirabili spettacoli. Il padrone, girando per la piazza in mezzo a una grande quantità di gente, di nascosto al cane toglieva agli spettatori gli anelli d'oro e d'argento o di ferro e, dopo averli deposti al suolo, li copriva con della terra. Quindi a un suo comando, il cane li prendeva a uno a uno e restituiva a ciascuno il suo. Allo stesso modo, tirava fuori a una a una e nominativamente delle monete di imperatori diversi, ammucchiate e confuse. Addirittura nel circolo degli astanti, il cane interrogato riconosceva senza sbagliare le donne gravide, i donnaioli, gli adulteri, gli avari e gli indigenti, e infine i generosi”. Cfr. L. SAGACIS, Historia Romana, a cura di Amedeo Crivellucci, Roma, I9II-I9I2, vol. II, pp. 5I-52. 
in pochi casi garantivano un reddito fisso e stabilito. Anzi, molto più spesso erano costretti a sollecitare continuamente una regalìa, una giubba, una cavalcatura.

Vi era tuttavia anche chi riusciva a ricavare sostanziose prebende e a vivere lautamente a spese dei suoi padroni. Questo era certamente il caso del fiorentino Dolcibene de Tori che nel 1355 fu incoronato re dei suoi pari da Carlo IV di Lussemburgo e che, grazie alle sue doti di faceto intrattenitore, godeva di un solido benessere economico, dal momento che si spostava su e giù per l'Italia accompagnato da un codazzo di cavalli e di famigli. Di lui il narratore toscano Giovanni Gherardi da Prato, ci dice che "in guadagno e sollazo vivea".

Sui modi con i quali Dolcibene si procurava denaro extra abbiamo una testimonianza davvero truculenta raccontata dal grande novelliere Franco Sacchetti, che peraltro era suo buon amico e dunque degno di fede. Racconta dunque il Sacchetti:

Nel tempo che messer Francesco degli Ardalaffi era signor di Forlì, una volta fra l'altre v'arrivò messer Dolcibene: e volendo il detto signore per esecuzione fare castrare un prete, e non trovandosi alcuno che 'l sapesse fare, il detto messer Dolcibene disse di farlo elli. Il capitano non averebbe già voluto altro, e così fu fatto. E messer Dolcibene fece apparecchiare una botte, e sfondata dall'uno de' lati, la mandò in su la piazza facendo là menare il prete, ed elli col rasoio e con uno borsellino andò nel detto luogo. Giunti là e l'uno e l'altro, e gran parte di Forlì tratta a vedere, messer Dolcibene avendo fatto trarre le strabule [= brache] al prete, lo fece salire su la botte a cavalcioni, e li sacri testicoli fece mettere per lo pertugio del cocchiume [=nel foro della botte]. Fatto questo, ed elli entrò di sotto nella botte, e col rasoio tagliata la pelle, gli tirò fuora, e misseli nel borsellino, e poi gli si mise in uno carniere, però che s'avvisò, come malizioso, di guadagnare, come fece. ${ }^{8}$

L'infame guadagno Dolcibene lo ottenne vendendo allo stesso prete per ventiquattro monete l'orribile trofeo, che nel frattempo aveva avuto l'accortezza di mettere sotto sale per conservarlo meglio. Questo perché lo sventurato, portandoli in tasca, non sarebbe rientrato nella categoria di coloro che non hanno i testicoli e avrebbe così potuto continuare a celebrare messa, assicurandosi la sopravvivenza economica, dal momento che testiculos qui non habet Papa esse non posset.

Un altro caso clamoroso di buffone decisamente benestante, ovvero, che avrebbe potuto essere addirittura ricco, se non fosse stato affetto da innumerevoli vizi, è relativo a Scocola, l'inquietante buffone del duca di Ferrara Borso d'Este. Lo vediamo in questo affresco [Fig. 2] dipinto, guarda caso, proprio mentre riceve una moneta da Borso. Costui aveva una famiglia composta da una moglie e dodici

7 Cfr. G. Gherardi, Il Paradiso degli Alberti, a cura di Antonio Lanza, Roma, I975, libro III, $\$$ I9O-I92, pp. 20I-202.

8 Cfr. F. Sacchetti, Opere, a cura di A. Borlenghi, Milano, I957, nov. xxv, pp. IO8-iIo. 
figli, ma non basta, aveva anche un servo e una fantesca a loro volta con numerosa prole. Bisogna davvero credere che i suoi lazzi fossero di gran qualità, perché il mantenere una simile tribù doveva essere di non lieve aggravio per le casse del ducato. Insomma, Scocola è sempre costretto a chiedere quantità incredibili di denaro al tesoriere di corte (giunge perfino a chiedere il corrispettivo del valore di una intera abitazione per pagare un debito di gioco) e l'incredibile è che riesce sempre ad ottenerlo, minacciando di volta in volta di essere costretto a trasferirsi altrove, oppure paventando di dover finire in ospedale per la prevedibile violenta reazione dei suoi creditori.

Concludiamo ora questo argomento sui guadagni dei giullari con un divertente passo del francescano fra' Salimbene da Parma dove vediamo in azione un vero campione di faccia tosta. Siamo al tempo dell'assedio di Parma del I248 e il protagonista passivo è il cardinale diacono Ottaviano, legato in Lombardia durante quei tragici avvenimenti:

Cum quadam die fieret quedam magna processio, et ipse transiret, dixit elevata voce eo audiente ioculator quidam: "Cedatis et removeatis vos de via, et permittite transire hominem, qui curie Romane proditor fuit et Ecclesiam frequenter decepit". Audiens hoc cardinalis precepit uni de suis silenter, ut clauderet os eius pecuniam sibi dando, sciens quoniam pecunie obediunt omnia. Et sic vexationem suam redemit, quia joculator in continenti donariis transtulit se ad alium locum, per quem cardinalis erat transiturus, et eum multicipliter commendavit dicendo quod in curia non erat cardinalis melior eo, et quod revera dignus esset papatu. ${ }^{9}$

Non sempre però i nostri amici attingevano il loro scopo. Con una certa frequenza i signori si divertivano a deludere le loro aspettative con scherzi di ogni tipo. Il letterato veneto Anton Francesco Doni [vissuto nella metà del xvi secolo] racconta una facezia che ha per protagonista "un greco molto ricco [...] che sempre fu nimico de' buffoni”. Un giorno si presenta da lui un buffone che

gli si fa incontro raccontandogli le sue sciocchezze. Il greco, che era astuto e sagace signore, prese quelle sue stoltizie per buone e care e gli disse: - Tu sia il ben venuto; quanto tempo

9 "Un giorno che si faceva una grande processione, mentre lui passava, un certo giullare disse a voce alta, che lui sentisse: -Fate largo e tiratevi indietro dalla strada! E lasciate passare un uomo che è stato traditore della corte romana e ha ingannato molte volte la Chiesa! - . Sentendo questo, il cardinale ordinò sottovoce a uno dei suoi che gli chiudesse la bocca dandogli del denaro: sapendo che al denaro tutto obbedisce. E così si liberò dalla sua persecuzione, perché il giullare, appena ricevuti i soldi, si trasferì immediatamente in un altro posto, dove il cardinale doveva passare, e lodò in molti modi il cardinale, dicendo che alla corte non c'era nessuno migliore di lui e che veramente era degno del papato". Cfr. Salimbene de Adam, Cronica, a cura di Ferdinando Bernini, Bari, 1942, p. 536 . 
è che io t'aspetto! Io voglio che tu stia qua seduto a capotavola; e per una volta io ti voglio far godere. - E gli fece vedere il pasto tutto preparato in tavola, gli fece assaggiare un vino prezioso e con un modo garbatissimo prese a dire: - Signori, voi conoscete la nostra usanza: prima di sederci a tavola, bisogna fare tre salti all'insù per poi poter meglio pranzare e poi tre salti in lunghezza; e chi vince il salto in alto ha il secondo posto a tavola, e chi vince il salto in lungo sta a capotavola: e io sarò stamattina il primo. - E, fatti tre salti in alto, vinse; dopo lui, saltò il buffone, e tutti gli altri. —Or su — disse il conte- egli mi tocca il secondo posto. Poi prese la rincorsa per il lungo della stanza e fece tre piccoli salti, tanto che arrivò fuor della porta mezzo braccio. Il buffone sùbito prese la sua rincorsa, per guadagnarsi il primo posto, e con tre salti quanto potette saltò; così egli uscí fuori più di due braccia. Il greco, che s'era fermato dentro all'uscio, facendo finta di vedere chi più saltava, quando lo vide fuori, chiuse la porta e lo lasciò là, dicendo: — Va, ché noi te la diamo vinta. —Così il buffone s'accorse d'essere stato imbrogliato. ${ }^{\text {Io }}$

Né ricchi né poveri erano dunque i nostri amici, bensì persone costrette a sbarcare il lunario barcamenandosi come meglio potevano nella sempre difficile navigazione della vita: oggi satolli e ben rimpannucciati e domani costretti a rigirarsi fra poveri stracci nelle gelide notti invernali, con lo stomaco che ruggiva per i morsi della fame.

I giullari, infatti, erano legati ai capricci e alle bizze dei loro padroni, poiché l'umore dei potenti è mutevole come una banderuola al vento. Ci soffermeremo su un solo caso, quello che riguarda il buffone milanese Tricano, detto Rastellino. Questi, nel I376, non potendo più reggere alle crudeltà di cui era fatto oggetto, si allontanò dalla corte milanese per rifugiarsi a Mantova presso i Gonzaga. Ma una lettera della cancelleria viscontea trasmette a quella gonzaghesca la preghiera di rinviare il poveretto a Milano nelle condizioni qui descritte:

Rogamus Fratern. V. quatenus placeat jubere ipsum ligatum subtus unius asini cum manibus retroligatis nobis trasmitti. ${ }^{\mathrm{II}}$

Una posizione niente affatto comoda, come si può facilmente comprendere, ove si pensi che il viaggio durava alcuni giorni.

Né è vero che soltanto i più abili fra loro fossero scelti dai signori del tempo per essere accolti nelle corti e mantenuti, poiché le mode e i gusti dell'epoca portavano spesso a scegliere per il ruolo di buffoni esseri infelici quali nani, gobbi, dementi, i quali, più che per il genio comico, facevano ridere i loro crudeli con-

Io Cfr. A. F. Doni, I marmi, a cura di E. Chiorboli, Bari, I928, p. Iv, "Il Nobile e il Perduto academici peregrini", p. 237-238.

II "Vi preghiamo fraternamente che vogliate ordinare che ci sia rimandato appeso sotto un asino con la mani legate dietro la schiena". Cfr. E. Levi, "L'ultimo re dei giullari", Studi Medievali, a. VI, vol. v, f. I, 2I (I928), p. I76-I77. 
temporanei per le deformità fisiche, per la loro stoltezza o autentica follia. E qui mi pare cada a proposito questo brano di una lettera scritta da Bernardino Prosperi a Isabella d'Este il 9 luglio I5I9. Vi si parla di una certa Caterina matta, nana di Lucrezia Borgia:

Credo che V. S. sapia de li modi et costumi suoi et che ogni pocho vino la rescalda et $\mathrm{fa}$ zornear come una bella regaza et ogni pocho più la fa furiosa de menare le mane e trar quanto ge viene inanci in faza et contra de chi se li trova senza riguardo alcuno et senza rispecto de persona, et dice quanto se li presenta alla bocha. Et se veruno ge dirà: -Passa l'acqua-, se alza li panni tonda sino dove po' et fa mostra di tuto il suo. ${ }^{\mathrm{I2}}$

La povera donna dunque, per timore di bagnarsi i vestiti, li sollevava ben oltre ove la decenza avrebbe consentito.

Il giullare di piazza è anch'esso, e a maggior ragione, legato ai capricci della fortuna; egli tuttavia, rispetto al buffone di corte, conduce una vita più autonoma, seppure più difficile, lontano dagli agi dei palazzi e delle tavole imbandite. La lotta per la sopravvivenza aguzza il suo ingegno. Inoltre in alcuni casi egli è in grado di rivendicare una significativa dignità artistica. Anche per loro poteva darsi il caso, invero eccezionale, che raggiungessero una certa agiatezza economica. Una conferma ci viene dalle parole del rimatore cremonese duecentesco Girardo Patecchio, che nel suo componimento più famoso, la Frotula noie moralis, si è preso la briga di elencare le cose spiacevoli che ci avvelenano l'esistenza; e fra queste non esita ad inserire anche il "rico ioculatore". ${ }^{13}$

Una specificità della giulleria italiana di cui ritengo utile dare conto, riguarda una particolare categoria di intrattenitori che fiorì in alcune comunità cittadine dell'Italia centrale a partire dal Trecento e fino alla metà del Cinquecento circa. Qui si diffuse l'uso di assumere e stipendiare da parte dei Comuni alcuni fra i più celebrati cantastorie dell'epoca e a loro si assegnò il compito di allietare i momenti di riposo dei gestori della cosa pubblica per distrarli dal peso dei loro gravi uffici. $\grave{E}$ chiaro che in tali circostanze queste figure finivano con lo staccarsi abbastanza nettamente da quelle classiche dei giullari di piazza, fino ad assumere una fisionomia loro propria in armonia col tipo di vita che conducevano.

A Firenze, l'incaricato di tale ufficio si chiamò "araldo della Signoria" (ma comunemente era detto anche "buffone" o "canterino") e la sua figura presto riunì in sé due ruoli distinti, quello di cavaliere di corte (milites curialis) e quello di sindaco e referendario del Comune. Il cavaliere di corte aveva l'incombenza di

I2 Cfr. A. Luzio \& R. Renier, "Buffoni, nani e schiavi dei Gonzaga ai tempi di Isabella d'Este”, Nuova Antologia, s. III, vol. XxxIV, I settembre I89I, p. II9.

I3 Cfr. E. Monaci, Crestomanzia italiana dei primi secoli, Nuova edizione riveduta e aumentata per cura di Felice Arese, Roma, I9552, p. I4O, v. 28. 
rallegrare onestamente la mensa dei Signori senza lazzi scurrili poiché, in quest'accezione, si può affermare che in Firenze il buffone non buffoneggiava. Quanto al sindaco e referendario, il nome chiarisce il compito, che era quello di riferire degli ufficiali prevaricatori e accusarli nei sindacati; a lui spettavano anche l'inventario, la custodia e il rinnovo delle masserizie e degli arnesi di palazzo.

Anche nella Bologna della prima metà del Quattrocento, per alleviare la quasi completa clausura degli Anziani, si stipendia un buffone che ha il compito di ricreare le loro eccellenze durante le pause del lavoro. ${ }^{14}$ Costoro, infatti, avevano il compito di governare la città restando reclusi nel Palazzo per i due mesi in cui durava il loro mandato. Una saggia disposizione giustificata dal desiderio di evitare qualsiasi tentativo di corruzione da parte dei cittadini.

A Perugia questi araldi si esibivano durante i pasti dei Priori due volte al giorno, de mano et de sero. In seguito si decise di offrire questo svago anche al popolo, dapprima con una certa misura, poi più largamente, cosicché ciò che inizialmente fu solo una graziosa concessione, in seguito diventò un obbligo del canterino e un diritto del popolo. Questa consuetudine riuscì assai gradita e così, di volta in volta, si stabilirono i giorni e le modalità in cui i canterini dovevano prestare la loro opera; essi dovevano: "cantare nei giorni festivi in estate nella piazzetta di santa Maria al Mercato, e in inverno nel palazzo del podestà". ${ }^{\text {s }}$

È stata l'Italia umanistica a fornire il liquido amniotico nel quale ha avuto la sua gestazione il complesso meccanismo che ha avviato la rinascita del teatro moderno. E qui, come altrove, i giullari e i buffoni di corte sono stati i protagonisti ineludibili di questi avvenimenti. Essi erano la scelta più ovvia per calcare le scene e impersonare i personaggi sacri e profani, tragici e comici perché erano "professionisti" riconosciuti e a loro si affidavano ruoli complessi e delicati. Le testimonianze che ho potuto raccogliere a questo proposito non sono numerose, ma mi paiono significative. Esse riguardano sia occasioni propriamente "teatrali", sia eventi festivi la cui complessa scenografia era senza dubbio un evento spettacolare assimilabile a una rappresentazione teatrale.

Cominciamo con un esempio risalente al secolo XI. Presso il Monastero di Farfa (località poco a Nord di Roma) si rappresentava la Coena Cypriani, che esigeva la presenza di due o almeno un istrione professionista. Afferma in proposito Vincenzo De Bartholomaeis:

\footnotetext{
I4 Cfr. F. Giorgi, "Un buffone degli Anziani di Bologna nel secolo xv”, L’Archiginnasio, xxiv, nn. I-3, (I929), pp. I20-I3O.

I5 Testo latino in A. D'Ancona, I canterini dell'antico Comune di Perugia, Varietà storiche e letterarie, I serie, Milano, I883, p. 48.
} 
Le parti di costoro erano, invero, le meno facili. Una era la parte del vocator. Il vocator, stando al di fuori del palco, veniva facendo l'appello de' personaggi, a misura che costoro si presentavano sulla scena, ciascuno col proprio attributo, o da soli ovvero in formazioni di cui egli spiegava il significato agli spettatori. L'altra era la parte del protagonista Gioele. Questi stava di continuo sulla scena: moderava e dirigeva l'azione, cioè il formarsi e lo sformarsi de' quadri plastici. ${ }^{16}$

Il buffone, attore e costruttore di meccanismi scenici di origine bolognese Ercole Albergati, detto "Zafarano", mentre prestava servizio alla corte del marchese di Mantova, viene richiesto a Milano nell'aprile I484 da Ludovico Maria Sforza: "per adoperarlo nella settimana santa a fare qualche rappresentazione, essendo lui aptissimo" ${ }^{17}$. Il Moro dunque specifica di volere proprio lui, perché considerato particolarmente adatto al compito che intende affidargli.

Questa graziosa immagine miniata da Pietro da Birago [Fig. 3] intorno al I495, ci mostra il nano del giovane Massimiliano Sforza che sembra guidare il corteo del carro trionfale su cui è seduto lo stesso futuro duca. Il nano indossa una sorta di "divisa" che contiene i colori della corte sforzesca, egli con la destra batte su un tamburo e con la sinistra suona il piffero. Anche in questo caso, è lui il vero protagonista della scena ed è giustamente posto in piena evidenza, lasciando in secondo piano perfino il duchino.

Nel I5I3 il celeberrimo buffone di Leone x, fra Mariano, fu chiamato a Firenze dal cardinale Giovanni de Medici (che colà si trovava per sottrarsi alla prigionia dei francesi) allo scopo di rallegrare il carnevale di quella città. Per l'occasione, infatti, erano stati organizzati "triomphi, comoedie et moresche", eventi spettacolari ai quali l'inesauribile verve comica del buffone avrebbe aggiunto non poco sapore. Ne abbiamo conferma da una lettera dello stesso frate, datata 29 gennaio e indirizzata al marchese di Mantova Francesco Gonzaga. Qui leggiamo che il buffone, se pure lascia trapelare la soddisfazione di poter tornare nella sua città natale, con notevole faccia tosta si lamenta per la fretta che gli è stata imposta, nonostante le sue, presunte, malferme condizioni di salute. Infatti, egli scrive: "Il mio padrone Monsignor de Medici mi costringe a muovermi in tutta fretta, nonostante io sia vecchio e mezzo malato". ${ }^{18}$

Due anni dopo, lo stesso Leone $\mathrm{x}$ riceve una straordinaria accoglienza durante un'altra visita nella sua città d'origine. Nel corso dei festeggiamenti vediamo un altro buffone romano, Mastro Andrea, che avanza trionfalmente su un superbo cavallo berbero curiosamente vestito e con le caratteristiche scarpe buffonesche

i6 Cfr. V. De Bartholomaeis, "Giullari farfensi”, Studi Medievali, vol. I, n. s. (I928), p. 45.

I7 Cfr. A. D’Ancona, Origini del teatro italiano, Roma, I89I², vol. II, p. 36I.

I8 Cfr. A. Luzio, "Federico Gonzaga ostaggio alla corte di Giulio II", Archivio della R. Società Romana di Storia Patria, vol. IX, fasc. III-IV, (I886), pp. 55I-552. 
oggi ereditate dai clowns del circo. Anche lui è parte integrante dell'iconografia tipica di queste occasioni:

Andrea buffone romano su un cavallo turcho con una zornea alla antiqua con la beretta rosa alla carmignola, li schinere di ferro in gamba: et uno par di scarpe con le punte lunghe mezzo brazo. ${ }^{19}$

Con questa graziosa immagine, mi congedo da voi ringraziandovi per l'attenzione.

I9 "Andrea buffone romano avanza su un cavallo turco indossando una mantelletta all'antica, un berretto rosso alla carmagnola, le gambe ricoperte da schiniere di ferro e un paio di scarpe con le punte lunghe mezzo braccio". Cfr. F. Chiericati, "Descriptione de la Entrata de la S.tà di N.S. papa Leone X.mo in la città di Fiorenza...", cit. in J. Shearman, "The Florentine Entrata of Leo X, ISIS", Journal of the Warburg and Courtauld Institutes, vol. 38 (I975), p. I. 


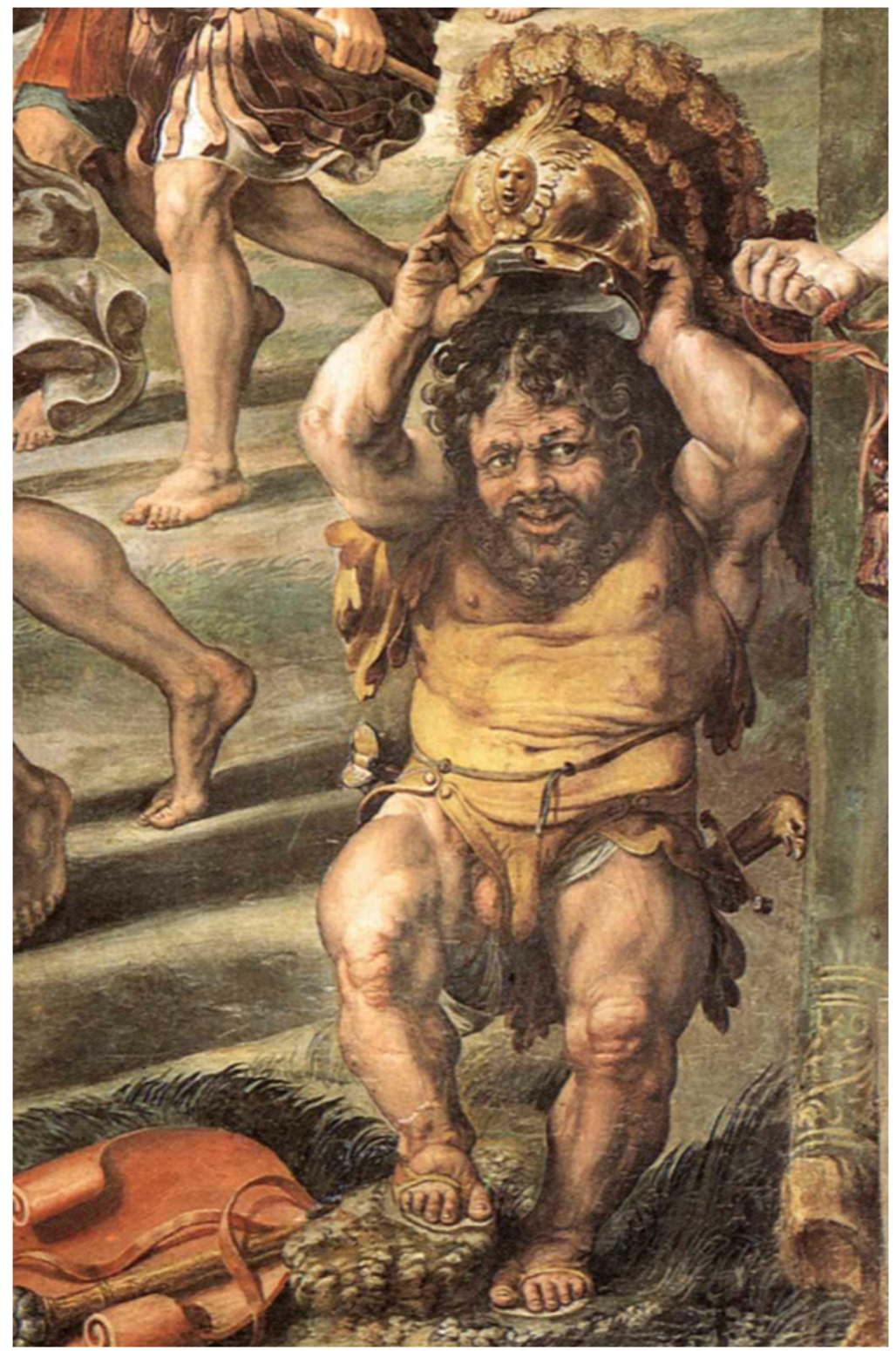

I. Giulio Romano [I499-I546] e collaboratori, Il nano Gradasso, particolare dell'affresco Visione della Croce nella Sala di Costantino in Vaticano. 


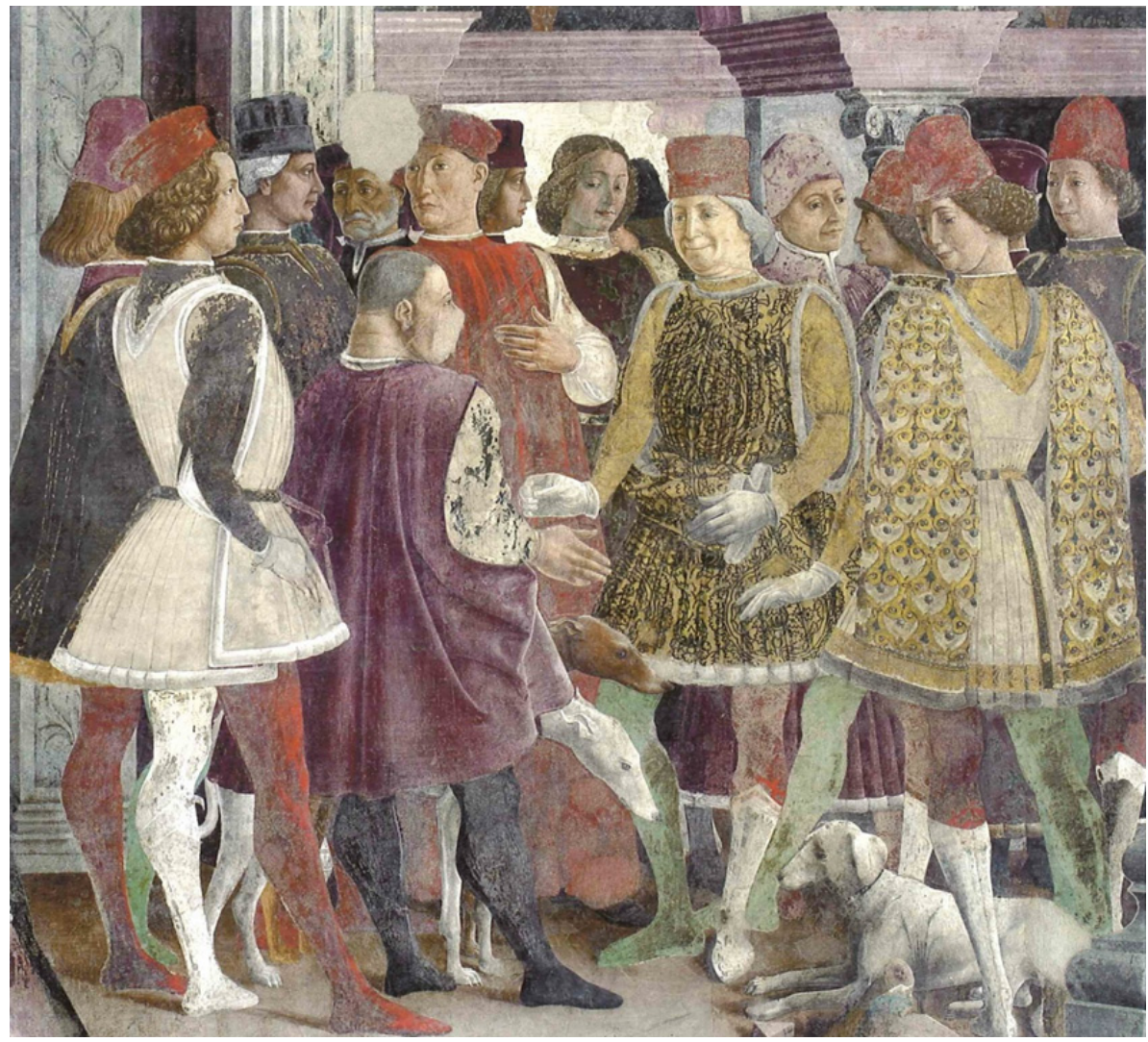

2. Francesco del Cossa (1436-78), Il duca Borso regala una moneta a Scocola, particolare dell'affresco del Mese di aprile, Ferrara, Palazzo Schifanoia. 


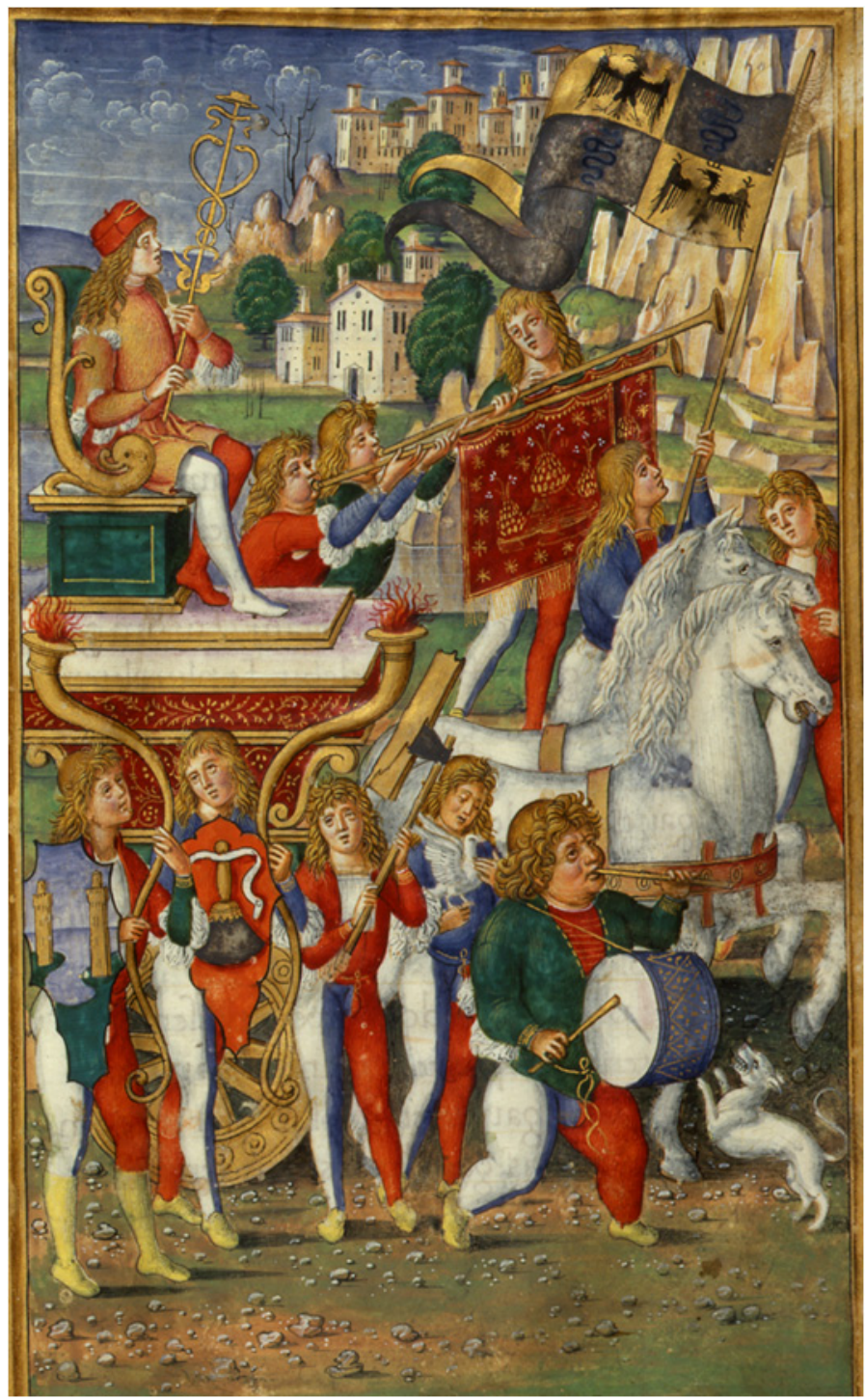

3. Pietro da Birago (I450 ca.-I513), Grammatica di Donato, Milano, Biblioteca Trivulziana, Ms. 2I67, fol. 29v. 